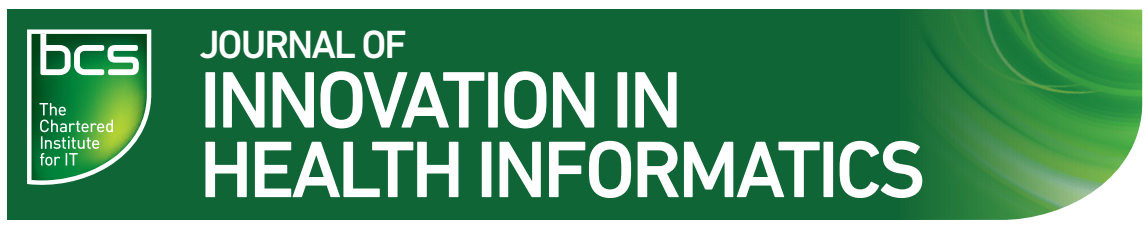

\title{
Practical applications and discussions
}

Cite this article: Freed J, Lowe C, Flodgren G, Binks R, Doughty K, Kolsi J. Telemedicine: is it really worth it? A perspective from evidence and experience. J Innov Health Inform. 2018;25(1):014-018.

http://dx.doi.org/10.14236/jhi.v25i1.957

Copyright ( 2018 The Author(s). Published by BCS, The Chartered Institute for IT under Creative Commons license http://creativecommons.org/licenses/by/4.0/

Author address for correspondence:

James Freed

Health Education England

Stewart House

32 Russell Square

London WC1B 5DN, UK

Email: james.freed@hee.nhs.uk

Accepted February 2018

\section{Telemedicine: is it really worth it? A perspective from evidence and experience}

James Freed

Health Education England, London, UK

Charles Lowe

Digital Health and Care Alliance, UK

Gerd Flodgren

National Institute of Public Health, Norway

Rachel Binks

Digital and Acute Care, Airedale NHS Foundation Trust, West Yorkshire, UK

Kevin Doughty

i-Centre for Usable Home Technologies, UK

Jyrki Kolsi

NHS Improvement, London, UK

\section{ABSTRACT}

Background This paper summarises a series of presentations on telemedicine given at a UK eHealth Week conference session in 2016. The formal evidence base for telemedicine is equivocal, but practical experience suggests that implementations of technology that support telemedicine initiatives can result in improved patient outcomes, better patient and carer experience and reduced expenditure.

Objective To answer the questions 'Is an investment in telemedicine worth it'? and 'How do I make a telemedicine implementation work'?

Methods Summary of systematic review evidence and an illustrative case study. Discussion of implications for industry and policy.

Results Realisation of telemedicine benefits is much less to do with the technology itself and much more around the context of the implementing organisation and its ability to implement.

Conclusion We recommend that local organisations consider deployment of telemedicine initiatives but with a greater awareness of the growing body of implementation best practice. We also recommend, for the National Health Service, that the centre takes a greater role in the collation and dissemination of best practice to support successful implementations of telemedicine and other health informatics initiatives.

Keywords: telecare, telemedicine, telehealth, health informatics, digital health, health technology, technology policy 


\section{INTRODUCTION}

As part of a recent UK eHealth Week conference, BCS Health \& Care hosted a session on telemedicine. This 90-minutes series of presentations was intended to give delegate answers to the questions 'Is an investment in telemedicine worth it'? and 'How do I make a telemedicine implementation work'? To answer these questions, the session was constructed to include a summary of the current evidence, a demonstration of recent case studies with real examples of learning, the policy position and the role industry can play in ensuring success.

Contributors to the session who had first-hand experience of implementing telemedicine interventions found it difficult to reconcile their experience with the findings of the Cochrane systematic review on the subject. The session was initiated to explore this apparent contradiction.

This paper is a summary of that session to support local decision makers investigating telemedicine. We hope that it gives health and care informaticians, clinicians and commissioners a reason to consider informatics innovations even when a systematic review appears to demonstrate equivocal benefit.

\section{THE EVIDENCE BASE}

Recent decades have seen a revolution in the field of technology, which has changed the way we interact with other people and also how we seek and exchange information. Importantly, information and communication technologies (ICTs) may also potentially revolutionise the delivery and organisation of healthcare in many different ways: improving health outcomes, reducing costs and increasing access, especially in underserved and rural areas and in low-income countries. However, it is important that the implementation of technology be accompanied by thorough evaluation that supports its effectiveness in specific clinical situations.

The Cochrane Effective Practice and Organisation of Care group conducted a systematic review of the literature to determine the effects of interactive telemedicine on professional practice and healthcare outcomes, ${ }^{1}$ when compared with traditional face-to-face delivery of care. The review, published in the Cochrane library in September 2015, included 93 randomised controlled trials (RCTs), in which telemedicine was used in direct patient care, either as a supplement, or as a substitute for usual care and assessed the effectiveness, acceptability and costs of interactive telemedicine. It did not assess the reasons why a telemedicine intervention may have demonstrated, or failed to demonstrate, significant benefits.

A majority of the included studies targeted patients with chronic conditions like heart failure and diabetes. In these studies, telemedicine was mainly used for remote monitoring of the condition, to enable prompt treatment and advice. In other studies, telemedicine was used to provide treatment, rehabilitation, education and advice for self-management or specialist consultations, to give some examples. For each condition, the review pooled outcome data that were sufficiently homogeneous. This limited the pooling of results to heart failure and diabetes outcomes.

The review findings indicate that the use of telemedicine in the management of heart failure may lead to similar health outcomes as face-to-face or telephone delivery of care. There is also evidence that telemedicine can improve the control of blood glucose in people with diabetes. The cost to health services, the acceptability by patients and healthcare professionals and the effects on professional practice are not clear due to limited data reported for these outcomes. Videoconferencing studies recruiting participants requiring mental health services, or specialist consultations for a dermatological condition reported no differences between groups. There was some evidence that remote monitoring could improve blood pressure control in participants with hypertension, while findings from the other studies were inconsistent.

There are a couple of limitations with the review to consider. First, the search cut-off may have resulted in the inclusion of outdated telemedicine systems. Second, it was impossible for the investigators to keep up with the large volume of trials continuously being published, wherefore a large number of potentially eligible studies had to be listed as awaiting assessment at the date of publication. Third, by including very small studies, the timeliness of the publication was hampered. ${ }^{2}$

Very few of the included studies contained data on organisational factors suggested to be important ${ }^{3}$ when new telemedicine programs are initiated, for example, the readiness of healthcare professionals to change their practice, the preparedness of the healthcare organisation and the time allowed for integration of telemedicine into a local health system.

Although there are gaps in our knowledge of what works in which conditions, there is reason for optimism. The large number of recently published and ongoing studies will add to the current evidence base. The increasing cell-phone coverage ${ }^{4}$ especially in low-income countries, and the fact that the next generation of older people will most likely be much more comfortable using ICT, adds to the optimism surrounding the future use of telemedicine.

\section{THE ART OF THE POSSIBLE - EXPERIENCES FROM AIREDALE}

Nearly 400,000 older people live in care homes in the United Kingdom, nearly $20 \%$ of whom are aged 85 and over. Most have complex health needs, including multiple long-term conditions, significant disability and advanced frailty. Collectively, they have high rates of both necessary and avoidable hospital admissions. ${ }^{5}$

Care provided to this complex cohort of people is often fragmented and services vary widely in standard, with high staff turnover and limited support from the wider health system. The Digital Care Hub at Airedale National Health Service (NHS) Foundation Trust (NHSFT) was opened in September 
2011 to provide $24 / 7$ clinical video assessment to support staff in caring for these residents as well as other patient groups. Having used telemedicine to deliver face-to-face consultations for patients in their own homes, the care home model was developed as it was thought to be more cost-effective, led by nurses and supported by the specialists in the acute trust. Airedale NHSFT had already been successfully providing remote outpatient consultations to prisoners since 2007, which had led to the prevention of unnecessary transport of offenders to hospital and had greatly reduced both the bed watch and escort budgets in those prisons using the service.

Discussions with nurses undertaking consultations indicated that they felt the three key aspects required for the telemedicine service to function well were: access to a clinical assessor; access to electronic patient records and the ability to clearly see the patient using a high-definition video link. The services at Airedale's Digital Care Hub have developed to include remote general practitioner (GP) triage, where care homes call the hub to assess the residents rather than calling the GP; the Gold Line, a telephone and video service to patients on the Gold Standards Framework ${ }^{6}$ thought to be in their last year of life; an Intermediate Care Hub, where health and social care staff work together to provide patients with the most appropriate service for their needs including a stepup and step-down pathway for frail elderly patients, before making the decision that they may need to reside in a care facility; and a complex care team that support those service users in the community with the highest reliance on health and social care. Patients and their carers have access to a clinical team $24 / 7 / 365$ which aims to promote their wellbeing, encourage self-care and enhance the use of informal support mechanisms, primary care and community services. Operational and clinical pathways have been implemented, working closely with community and primary care teams, to enable early intervention and timely identification and support for patients, particularly those residing in care homes leading to a reduction in inappropriate attendance at the emergency department (ED) or acute admission to hospital. ${ }^{7}$ The use of remote video consultation has also demonstrated a reduction in GP visits (particularly out of hours), and the need for numerous community team and District Nurse visits (Vanguard evaluation report in preparation).

It took a number of years to establish the digital care hub at Airedale NHSFT as commissioners were reluctant to contract a service without a strong evidence base and a critical mass of service users was necessary to develop this. A number of Clinical Commissioning Groups (CCGs) wanted to pilot the service in a small number of care homes, but this left the majority continuing to send residents to ED as they always had, making a significant outcome difficult to demonstrate. ${ }^{8}$

Clinicians were slow to adapt to this new way of working and questioned the governance around remote assessment which led to a nurse led service being developed which has proven to be both more cost effective and more robust across a 24/7 provision. Some care homes - particularly independent providers - needed to be persuaded that the hub would be beneficial for them. Airedale addressed this challenge by working with each care home to understand their difficulties and show how access to the hub staff could help them to better deal with their own problems. For example, the hub supports the staff to deliver better care to their residents, making the NHS Care Quality Commission (CQC) inspection regime easier and more transparent, and increasing staff's confidence and skills.

There were few technology providers available for the development phase. Airedale has a joint venture partner Involve - which supplies and installs a secure video link to the hub via a secure N3 (NHS network) platform. Each care home or patient needs to have access to at least one laptop and a reliable internet or $3 \mathrm{G} / 4 \mathrm{G}$ connection and they also have access to a 24/7 helpline for technical support. Cisco webcams, software and infrastructure are in use in Airedale, but any HD 1080p (hardware encoder) camera would be suitable. The $24 / 7$ technical support helpline is provided by Involve.

Despite numerous visits to the hub by interested trusts and commissioners, there are few other facilities set up across the country. This may be due in part to the financial investment required to deploy the infrastructure but also to the 24/7 staffing requirement and culture change needed to change from face to face to remote clinical assessment.

\section{THE ROLE OF INDUSTRY - PRODUCTS AND SERVICES}

Telemedicine and other products and services that enable medical/social care functions to be performed remotely are dependent on several technologies. These include the following:

- computing power,

- advanced analytics (software),

- intuitive device interfaces,

- standardised connectivity and communication formats

- sensors and other point of care devices,

- large data transfers, and

- secure storage, retrieval and display of data

Processing power and speed can be measured, but there are few objective ways of tracking improvements in the other technology elements. However, telecommunication speed is a particularly important factor for facilitating remote services; individual developments, especially in mobile technologies, are arguably the most significant motivators for supporting innovation, especially for people outside the hospital environment.

It may be apparent that new technologies and tele-services quickly follow each improvement in telecommunication bandwidth. The half-life of each new device must also reduce, along with opportunities to demonstrate, from a clinical perspective, successful outcomes such as efficacy and value for money. From a commercial perspective, it impacts the likelihood of achieving an acceptable return on investment, compromising incentives to innovate and preventing the 
transformation of service delivery needed to make health service more efficient, while reducing the demand.

There is a need to streamline processes of acceptance for introducing, and mainstreaming, new products and processes, but without compromising the need for evidence that both clinicians and the service commissioners rely on. The gold standard randomised control trial is inappropriate for remote and self-monitoring from a commercial point of view because

- most remote monitoring solutions are produced by small to medium enterprise (SMEs) who simply do not have the resources,

- many solutions support people that are without a formal diagnosis of an illness, so compromising timely participant identification,

- the market for many products will be small making it difficult to recruit sufficient numbers of participants,

- individual technologies are not designed to replace medical interventions, but are usually proposed as components in care plans, making their success dependent on other parts of the system that are difficult to control, and

- they often involve lifestyle changes making adherence to trial protocols difficult over the long periods required to confirm behavioural change and outcomes.

In order to support a more rapid route to market for remote monitoring and management strategies, service/product providers need stronger links to both the clinicians who can champion their products and to the service commissioners. All stakeholders need access to current evidence and usage data. Much of the evidence will be derived from end-users (i.e. patients) rather than from clinicians, which needs to be collected quickly and managed in a way that is trusted. As access to community equipment and telecare services becomes more restricted, giving way to more individually funded schemes (personal budget), the public will also need reliable and impartial advice to avoid them buying the devices that are low cost but lacking in the usage evidence that would confirm appropriateness and safety.

\section{THE ROLE OF POLICY - THE IMPACT OF PAYMENTS}

The nationally set prices and rules for local pricing that govern how money flows between commissioners and providers of NHS funded services should not be a barrier to innovation, including the effective use of telemedicine. Unfortunately, in practice, it is often felt to be just that, even if the rules themselves are flexible to at least accommodate it and at best enable it.

The national prices set for the Healthcare Resource Group (HRG) - standardised bundles of types of clinical activity are designed to act as a benchmark to beat. This should, in principle, enable and incentivise cost-effective innovations. However, often a particular set of resource and procedure codes need to get ticked for care to qualify for payment under the HRG, so the HRGs are either not available or relevant for new innovative ways of delivering care such as telemedicine.

There are two main ways in which the prices and rules at the moment accommodate and enable innovation:

- The local pricing rules, applied where there are no national prices or HRGs, are built on three principles that support innovation. The local payment approaches need to: ${ }^{9} 1$ ) be in the best interest of patients, 2) promote transparency and improve accountability and 3 ) be developed through constructive engagement.

- The payment rules also give explicit permission to agree to vary from the nationally set prices and HRGs. The locally agreed approach needs to satisfy the above principles, but there is no approval process to go through.

Using the local pricing rules can mean providers and commissioners need to put in extra effort. Developing a set of national prices for telemedicine would not be an effective solution to reduce the local burden. There are a multitude of ways and circumstances where telemedicine is used, and new ones are being trialled and implemented constantly. Prices would likely be out of date even before they were ready to be published. Worse prices for specific ways of doing telemedicine could then act to standardise provision before the best solutions have been discovered.

Instead, NHS Improvement is working to make the payment system more supportive of innovation and service reforms in all their forms. That includes work towards better cost, quality and activity data as well as new payment approaches focused on value to support new models of care. ${ }^{10}$ Paying provider groups that use capitation linked to outcomes (rather than activity) supports improvement in a number of areas:

- Predictability of upfront payment gives providers stability to plan and implement changes.

- Accountability and flexibility to meet the care needs of the population, coordinating across traditional boundaries, provides greater opportunities for changing care delivery across full pathways without immediate top line revenue impacts from changing types and amounts of activity.

- Risk transfer that rewards providers for doing the right thing, without a detailed specification of how to deliver care.

\section{DISCUSSION}

On the face of it, the evidence is confusing. Organisations small and large are clearly achieving significant benefit from using technology to support the modernisation of care delivery, Airedale being the best single example quoted. On the other hand, the evidence from RCTs is at best mixed.

Could it be that the problem is actually with the RCTs? They are designed as they are to check on the efficacy of medicines which have very different characteristics: 
- Many medicines are affected by the genetics of the person taking them;

- Medicines are clearly dependent on whether they are taken as prescribed (in the world, $50 \%$ are not, for a wide variety of reasons);

- As medicine relies mainly on chemical reactions, it is typically relatively independent of care pathway (within limits, of course);

- Medicines need regular replenishment;

- Telemedicine on the other hand is not genetically dependent;

- Failure of a user to send in vital signs, readings or other interactions is immediately evident, enabling remedial action to be taken;

- Most importantly, telemedicine delivers virtually all its benefits by enabling new models of care and so if constrained by an RCT that looks only at adding telemedicine without also allowing a change in care pathway is almost guaranteed to show little if any benefit - possibly even a net cost.

As a result of the above, perhaps it is now time to look at other ways of measuring the benefits of telemedicine using engineering principles - as Jeremy Wyatt has commented 'no one does an RCT of bridges', yet most of them deliver as planned a calculated level of benefit.

The Cochrane systematic review, and several studies published since (and notably the Airedale experience summarised here) demonstrate that telemedicine can provide significant benefits - to patient and carer experience, to support improved health outcomes and to reduce costs. Yet these benefits are not guaranteed.

\section{REFERENCES}

1. Flodgren G, Rachas $A$, Farmer $A J$, Inzitari $M$ and Shepperd S. Interactive telemedicine: effects on professional practice and health care outcomes. Cochrane Database of Systematic Reviews 2015;(9):CD002098. doi: 10.1002/14651858.CD002098.pub2. Available from: https://doi. org/10.1002/14651858.CD002098.pub2.

2. Rada G. Telemedicine: are we advancing the science? [editorial]. Cochrane Database of Systematic Reviews 2015;(9). doi: 10.1002/14651858.ED000105. Available from: https://doi. org/10.1002/14651858.ED000105.

3. Hendy J, Chrysanthaki T, Barlow J, Knapp M, Rogers A, Sanders $\mathrm{C}$, et al. An organisational analysis of the implementation of telecare and telehealth: the whole systems demonstrator. BMC Health Services Research 2012;12:403. Available from: https://doi.org/10.1186/1472-6963-12-403. PMid:23153014; PMCid:PMC3532839.

4. World Health Organisation. mHealth: New Horizons for Health Through Mobile Technologies: Second Global Survey on eHealth. Geneva, Switzerland: World Health Organization, 2011.
While the technology can provide benefits, it is the context in which the technology is implemented and the skill with which the implementation is conducted that realises benefits.

\section{CONCLUSION}

To answer the question posed in our title, the evidence demonstrates that telemedicine as a technology is worth it, although return on investment varies with the care pathway and, most significantly, in the ability of the team to implement effectively. However, given the huge dependence on context, including ability to implement, readiness of staff and patients to change and clear leadership, our conclusion is that telemedicine, and by extension health technology, will always be dogged by equivocal results because of the huge impact of context, outwith anything to do with the technology per se.

If that conclusion is accepted, two recommendations fall out. First, local implementers must concentrate on the science of implementation - the things to do and things to avoid, learning from others, both successful and unsuccessful. Examples of places to find these answers will be from authors of peer-reviewed papers, discussion forums [e.g. the Digital Health Intelligence Chief Information Officer ( $\mathrm{ClO})$ forum, the Digital Health \& Care Alliance or the CHAIN network] and professional champions. Second, policy makers and national funders should concentrate on making implementation advice more accessible and consumable through custom advice services, passive resources (such as the NHS Digital website) and by making resources available for free through other bodies, for example, the Federation of Informatics Professionals website or the e-Learning for Health portal.

5. Briggs R, Coughlan T, Collins R, O'Neill $D$ and Kennelly SP. Nursing home residents attending the emergency department: clinical characteristics and outcomes. Quarterly Journal of Medicine 2013;106:803-8. Available from: https://doi. org/10.1093/qjmed/hct136. PMid:23818688.

6. Available from: http://www.goldstandardsframework.org.uk/. Accessed December 2016.

7. Available from: https://www.gov.uk/guidance/moving-healthcare-closer-to-home. Accessed Decemebr 2016.

8. Hex N. Evaluation of telehealth interventions for care homes in Airedale, Wharfedale and Craven. YHEC, May 2015.

9. Section 6 in: Available from: https://www.gov.uk/government/ publications/nhs-national-tariff-payment-system-201617. Accessed December 2016.

10. Available from: https://www.gov.uk/government/collections/ different-payment-approaches-to-support-new-care-models. Accessed December 2016. 